\title{
Transferências de gestantes e recém-nascidos em um Centro de Parto Normal
}

\author{
Transfers of pregnant women and newborns in a Normal Birth Center \\ Traslados de gestantes y recién nascidos em um Centro de Parto Normal
}

Camila Prado dos Santos Mendonça ${ }^{1}$, Viviane Maria Gomes de Araújo ${ }^{1}$, Joanna Francyne Silva de Barros $^{1}$, Raine Danyele Vieira de Sousa ${ }^{1}$, Bárbara Bruna Fernandes de Andrade ${ }^{1}$, Monique Maria de Souza Baltar ${ }^{1}$, Fernanda Celiberti Soveral ${ }^{1}$, Taita Paloma Alves Diniz ${ }^{1}$, Thacia Bezerra Ferreira de Oliveira ${ }^{1}$, Camila de Mattos Oliveira ${ }^{1}$.

\section{RESUMO}

Objetivo: Apresentar o índice de transferências das mulheres e recém-nascidos, seus motivos e os desfechos dos partos de gestantes em um Centro de Parto Normal (CPN). Métodos: Estudo descritivo, transversal, exploratório e quantitativo. Foi desenvolvido no período de dezembro de 2019 a dezembro de 2020 em um CPN de uma cidade de Pernambuco. Teve uma amostra de 586 parturientes. Resultado: $27,4 \%$ das parturientes foram transferidas, sendo que $91,9 \%$ no intraparto e $4,9 \%$ no pós-parto. Os motivos mais prevalentes foram presença de mecônio no líquido amniótico e distócia de progressão. 54,4\% das transferências resultaram em parto normal. Já com relação aos recém-nascidos houve uma taxa de transferência de $44,7 \%$ e o motivo mais predominante foi icterícia, podendo estar relacionada ao clampeamento tardio de cordão umbilical, porém não se pode afirmar tal fato. Conclusão: Apesar das taxas de transferência maternas e neonatais neste estudo serem consideradas maiores que nos estudos analisados, não houve nenhum agravamento ou óbito, e em relação ao total de admissões, a taxa de cesariana encontrou-se dentro dos índices preconizados pela Organização Mundial de Saúde, sugerindo que a assistência em CPN pode ser segura para as gestantes de risco habitual.

Palavras-chave: Centro de parto normal, Transferência de paciente, Gestantes, Recém-nascido.

\begin{abstract}
Objective: To present the transfer rate of women and newborns, their reasons and the outcomes of the deliveries at a Birth Center. Methods: Descriptive, cross-sectional and exploratory study with a quantitative approach. It was developed from December 2019 to December 2020 at a Birth Center. There was a sample of 586 parturients. Result: $27.4 \%$ of the parturients were transferred, $91.9 \%$ in the intrapartum and $4.9 \%$ in the postpartum. The most prevalent reasons were the presence of meconium in the amniotic fluid and progression dystocia. $54.4 \%$ of transfers resulted in normal birth. Regarding the newborns, there was a transfer rate of $44.7 \%$ and the most prevalent reason was jaundice, which may be related to late clamping of the umbilical cord, however, this fact cannot be stated. Conclusion: Although maternal and neonatal transfer rates in this study are considered higher than in the studies analyzed, there was no worsening or death, and in relation to the total number of admissions, the cesarean rate was within the rates recommended by World Health Organization, suggesting that Birth Center assistance can be safe for pregnant women at usual risk.
\end{abstract}

Key words: Birth center, Patient transfer, Pregnant women, Newborn.

\footnotetext{
${ }^{1}$ Instituto de Medicina Integral Professor Fernando Figueira (IMIP), Recife - PE.
}

*E-mail: camilapradods@gmail.com

SUBMETIDO EM: 2/2021

ACEITO EM: 3/2021

PUBLICADO EM: 4/2021 


\section{RESUMEN}

Objetivo: Presentar el índice de transferencia de mujeres y RN, sus motivos y los resultados de los partos de gestantes en un Centro de partos normales (CPN). Métodos: Estudio descriptivo, transversal, de carácter exploratorio y enfoque cuantitativo. Se desarrolló de diciembre de 2019 a diciembre de 2020 en el a Centro de partos normales. La muestra fue de 586 mujeres embarazadas. Resultados: El 27,4\% de las mujeres fueron trasladadas, el $91,9 \%$ en el intraparto y el $4,9 \%$ en el postparto. Los motivos más frecuentes fueron la presencia de meconio en la fuido amniotico y la distocia por progresión. El 54,4\% de los traslados dieron lugar a un parto normal. En cuánto a los recién nacido, hubo una tasa de transferencia del $44,7 \%$ y el motivo más predominante fue la ictericia, que puede estar relacionada con el clampeo tardio Del cordón umbilical, pero no se puede afirmar este hecho. Conclusión: Aunque las tasas de transferencia materna y neonatal en este estudio se consideraron más altas que en los estúdios analizados, no hubo empeoramiento ni muerte, y en relación con el total de ingresos, la tasa de cesáreas estuvo dentro de las tasas recomendadas por la OMS.

Palabras clave: Centro de partos normales, Traslado de pacientes, Embarazada, Recién nacido.

\section{INTRODUÇÃO}

Os Centros de Parto Normal (CPN) são unidades destinadas a prestar assistência ao parto normal de risco habitual, ou seja, sem complicações obstétricas. Essa unidade contribui para a redução das taxas de cesáreas, uma vez que um de seus objetivos é assegurar à mulher o direito a ambientes de cuidado que favoreçam a realização das boas práticas de atenção ao parto e nascimento (BRASIL, 2015; GARCIA LV, et al., 2017).

O CPN oferece um atendimento humanizado e alinhado com as recomendações da OMS, estimulando à autonomia da parturiente e reduzindo práticas medicamentosas, intervenções desnecessárias e complicações puerperais. Tais práticas além de gerarem um melhor prognóstico para as mães e para os recém-nascidos, proporcionam uma maior satisfação das pacientes pela assistência recebida (GARCIA LV, et al., 2017).

Segundo estimativas, cerca de $20 \%$ de todas as gestações podem evoluir de forma desfavorável, tanto para o feto como para a mãe, configurando uma gestação de alto risco. Porém, a grande maioria leva um processo de gestação e parto sem intercorrências, seguindo com gestações de risco habitual ou baixo risco. Nos casos em que não há necessidade de se utilizar alta densidade tecnológica em saúde e nos quais a morbidade e a mortalidade materna e perinatal são iguais ou menores do que as da população em geral, as gestações podem ser consideradas como de baixo risco (RODRIGUES ARM, et al., 2017; BRASIL, 2012; BRASIL, 2012).

Estas pacientes, elegíveis para atendimento nos CPN, obedecem a critérios específicos que as caracterizam desta forma, visto que a assistência é prestada exclusivamente pelas enfermeiras obstetras. Mulheres portadoras de síndromes hipertensivas, diabetes, infecções ativas ou sem tratamento adequado, são alguns exemplos de condições impeditivas para admissão em um CPN, como também a necessidade de indução ou analgesia, pois é necessário que, nesses casos, sejam acompanhadas por um profissional médico (RODRIGUES ARM, et al., 2017; BRASIL, 2012).

Segundo a Organização Mundial de Saúde (OMS) (1996), os enfermeiros obstetras e obstetrizes são os profissionais mais adequados para o acompanhamento das gestações e partos de risco obstétrico habitual. Em 2016, a Cochrane publicou uma revisão que concluiu que, comparadas àquelas atendidas em outros tipos de modelos, as gestantes atendidas em um modelo de assistência obstétrica contínua liderado por obstetrizes tiveram menor probabilidade de sofrer intervenções, maior probabilidade de se sentirem satisfeitas com os cuidados recebidos e uma probabilidade semelhante de terem desfechos adversos maternos ou perinatais (SANDALL J, et al.,2016).

Apesar dos critérios estabelecidos para o internamento em um CPN, durante a assistência pode ser identificado algum fator de risco, e diante desse cenário, recomenda-se a transferência das pacientes para 
um setor de médio ou alto risco, onde serão acompanhadas por profissionais médicos. Quando o CPN é extra-hospitalar ou autônomo, é necessário que haja um hospital de referência para eventuais remoções (LIMA DM, et al., 2018).

De acordo com a lei 7.498 de 25 de junho de 1986, além da assistência à parturiente e ao parto normal de risco habitual, cabe ao enfermeiro a identificação de distócias obstétricas, complicações neonatais e a tomada de providências até a chegada ao médico (BRASIL, 2015).

Neste sentido, objetivou-se apresentar o índice de transferências das mulheres e RN, seus motivos e os desfechos dos partos de gestantes atendidas em um Centro de Parto Normal em Pernambuco. Assim, os resultados de estudos sobre remoções maternas e neonatais de centros de parto são de grande importância para a consolidação e eficácia deste tipo de serviço, trazendo benefícios para as mulheres e seus bebês.

\section{MÉTODOS}

Trata-se de um estudo descritivo transversal, com caráter exploratório e abordagem quantitativa. Foi desenvolvido no período de dezembro de 2019 a dezembro de 2020 em um Centro de Parto Normal (CPN) em Pernambuco, uma instituição filantrópica referência no atendimento materno-infantil no âmbito do SUS, e vinculado ao Programa de Residência em Enfermagem Obstétrica.

A população do estudo foi constituída pelas parturientes admitidas no CPN no período de Agosto de 2018 a Agosto de 2019, sendo uma amostra de 586 parturientes. A coleta de dados foi realizada por meio da análise dos dados registrados na planilha e livros de registro existentes nos setores, sobre as mulheres e recémnascidos. Foi utilizado um formulário confeccionado pela autora de acordo com os indicadores disponíveis nos livros e planilhas pesquisadas.

Essa pesquisa está integrada a pesquisa Perfil da assistência ao parto e nascimento em um centro de parto normal do nordeste brasileiro, submetida ao Comitê de Ética e Pesquisa com Seres Humanos do Instituto de Medicina Integral Professor Fernando Figueira (IMIP), sob CAAE: 21388919.8.0000.5201 de acordo com as resoluções $466 / 12$ e 510/16 do conselho nacional de saúde para pesquisa envolvendo seres humanos.

\section{RESULTADO E DISCUSSÃO}

No período do estudo, houve um total de 586 admissões de gestantes em trabalho de parto, e dessas, 161 $(27,4 \%)$ resultaram em transferências para o setor de alto risco sendo que $148(91,9 \%)$ no período intraparto, $8(4,9 \%)$ no período pós-parto e $5(3,1 \%)$ dos casos não havia informação disponível.

Tais achados mostram que o índice de transferência materna é superior aos encontrados em outros estudos envolvendo CPN e casa de parto, onde os índices de transferências variaram entre 0,7 e $12,3 \%$ (MENDES AS, et al., 2017; PEREIRA ALF, et al., 2013; PEREIRA ALF, et al., 2012; BONADIO IC, et al., 2011; SILVA FMB, 2011).

Somente 1 entre os estudos encontrados, evidenciou um índice maior de transferência, 31,5\% (OLIVEIRA TCM, et al., 2019). Tal diferença pode ser justificada pelo CPN do IMIP ter sido avaliado no seu primeiro ano de funcionamento, período este que ocorreram ajustes dos critérios de admissão e adaptação das equipes envolvidas com a assistência.

Outro fator que pode estar associado a um maior índice de transferência materna é o alto percentual de internamento no CPN por profissionais médicos, em alguns casos realizados fora do período de trabalho de parto ativo, o que pode acarretar mais tempo no serviço aumentando a taxa de cesariana.

Em todos os estudos, mais de $80 \%$ das mulheres foram transferidas no período intraparto, o que demonstrou uma característica semelhante ao presente estudo (OLIVEIRA TCM, et al., 2019; MENDES AS, et al., 2017; PEREIRA ALF, et al., 2012; BONADIO IC, et al., 2011; SILVA FMB, et al., 2011) (Tabela 1). 
Tabela 1 - Prevalência das transferências das gestantes e características das mães dos RN transferidos, $\mathrm{n}=161$.

\begin{tabular}{lcc}
\hline Período de transferência da gestante & № & $\%$ \\
\hline Período & 148 & 91,9 \\
Intraparto & 8 & 4,9 \\
Pós-parto & 5 & 3,1 \\
Sem informação & 161 & - \\
\hline Total &
\end{tabular}

Fonte: Mendonça CPS, et al., 2021.

A Tabela 2 apresenta as características das gestantes transferidas, e em relação ao perfil obstétrico, a única variável encontrada nos objetos de pesquisa foi a quantidade de gestações anteriores, e como resultado, 52\% das pacientes eram nulíparas. Silva FMB (2011), fala que a nuliparidade é apresentada como um fator de risco para a transferência, pois na maioria dos estudos encontrados, mais de $50 \%$ das transferências ocorreram nas primigestas.

Tabela 2 - Perfil obstétrico e demográfico das gestantes transferidas, $\mathrm{n}=161$.

\begin{tabular}{lcc}
\hline Características Obstétricas e Sociodemográficas & № & $\%$ \\
\hline Gestações & & \\
\hline Primeira gestação & 84 & 32 \\
Segunda gestação ou mais & 20 & 12,4 \\
Sem informação & & \\
\hline Idade & 34 & 21,1 \\
\hline 10 a 19 anos & 103 & 63,9 \\
20 a 35 anos & 6 & 3,7 \\
> 35 anos & 18 & 11,1 \\
Sem informação & & \\
\hline Estado civil & 96 & 59,6 \\
\hline Sem companheiro & 59 & 36,6 \\
Com companheiro & 6 & 3,7 \\
Sem informação & & \\
\hline Ocupação & 95 & 59 \\
\hline Não remunerada & 23 & 14,2 \\
Remunerada & 43 & 26,7 \\
Sem informação & & \\
\hline Município de residência & 140 & 86,9 \\
\hline RMR & 6 & 3,7 \\
Interior & 3 & 1,8 \\
Outro estado & 12 & 7,4 \\
Sem informação & & \\
\hline Fon : Mencha & & \\
\hline
\end{tabular}

Fonte: Mendonça CPS, et al., 2021.

Um estudo descritivo, com coleta retrospectiva, realizado em um Centro de Parto em São Sebastião, comparou os resultados das transferências, e nulíparas foram removidas em maior número (63,5\%) comparadas às multíparas (36,5\%) (MENDES AS, et al., 2017). Em uma casa de parto, nos anos entre 1998 e 2008, reportou uma taxa de transferência quase 4 vezes maior entre as nulíparas, quando comparadas as multíparas (BONADIO IC, et al., 2011). 
Esse grupo de gestantes apresenta uma maior taxa de remoções devido a maiores chances de implicações adversas como trabalho de parto mais prolongado e cesárea a pedido, o que também pode estar relacionado com a menor tolerância da mulher a situação do trabalho de parto ou insegurança sobre o processo.

Constata-se também que a idade da maioria das mulheres transferidas estava entre 20 a 35 anos $(63,9 \%)$ e $21,1 \%$ eram consideradas adolescentes e mostrou-se com resultados semelhantes a outros estudos nacionais, onde as adolescentes variaram entre 17,3 e 27,5\% (SILVA FMB, et al., 2011; BONADIO IC, et al., 2011; MENDES AS, et al., 2017). 63,9\% das pacientes transferidas não faziam partes dos extremos da idade reprodutiva, estando entre 20 e 35 anos.

Pacientes ditas sem companheiro constituíram o maior grupo das transferidas (59,6\%), onde se enquadram as solteiras, divorciadas ou viúvas, o que divergiu dos outros estudos sobre remoções em 2011, onde mais de 70\% relataram a existência de parceria (SILVA FMB, et al., 2011; BONADIO IC, et al., 2011).

De acordo com os estudos pesquisados que constaram esses dados, mais da metade das pacientes transferidas não exerciam atividade remunerada, que também foi evidente no presente estudo (59\%) (SILVA FMB, et al., 2011; BONADIO IC, et al., 2011; MENDES AS, et al.,2017; PEREIRA ALF, et al., 2013).

Com relação ao município de residência, percebe-se que a grande maioria tem como seu local de Residência a Região Metropolitana do Recife, 86,9\% e somente 3,7\% afirmaram morar no interior do estado. Entre os estudos, há uma média de $72,1 \%$ de pacientes que residem na área de abrangência do serviço, semelhantes aos dados que foram encontrados (SILVA FMB, et al., 2011; BONADIO IC, et al., 2011; MENDES AS, et al., 2017). Muitos Centros de Parto Normal têm como pré-requisito para o atendimento fazer parte da área de abrangência, porém o CPN do IMIP recebe e atende pacientes em demanda espontânea.

Para as remoções intraparto das gestantes relatados na tabela 3 , os motivos mais frequentes foram mecônio (8,6\%), distócia de progressão (8,6\%), síndromes hipertensivas $(7,4 \%)$, alteração de BCF $(6,8 \%)$, febre intraparto $(2,4 \%)$, sífilis $(1,8 \%)$ e infecção do trato urinário $(1,8 \%)$. Os outros motivos registrados não foram descritos na tabela pois não passaram de $2 \%$ da porcentagem, são eles: hemorragia pós-parto (2), prolapso de cordão (2), drogadição (2), macrossomia (2), diabetes gestacional (2), pródromos (2), centralização fetal (2), anidrâminio (1), prematuridade (1), analgesia (1), desproporção céfalopélvica (1), cesárea a pedido (1), plaquetopenia (1), placenta baixa (1), ausência de exames de PN (1), hepatite B, HIV (1), surto psiquiátrico (1). Ressalta-se que não foram encontrados nos registros os motivos de transferência de $46,5 \%$ mulheres (Tabela 3 ).

Com relação aos motivos no pós-parto, evidenciou-se: correção de laceração (1,2\%), febre pós-parto $(0,6 \%)$, curetagem $(0,6 \%)$ e retenção placentária $(0,6 \%)$. Durante a coleta foi encontrado uma realização de histerectomia total após o caso descrito de retenção placentária.

Dois estudos realizados na casa de parto David Capistrano Filho, no Rio de Janeiro, nos anos de 2012 e 2019, apontaram uma taxa de remoção materna de 12,3 e $31,5 \%$, respectivamente. Como observado, em sete anos houve um aumento significativo na porcentagem de transferências, porém os dois motivos mais prevalentes foram iguais, sendo eles: bolsa rota 33 e 50\% e mecônio 22,5 e 17,8\% (PEREIRA ALF, et al., 2012; OLIVEIRA TCM, et al., 2019).

Um estudo descritivo, em um CPN peri-hospitalar do Distrito Federal mostra que as principais causas de transferências intraparto foram: parada de progressão (34,6\%), alteração de BCF (17,3\%) e em terceiro lugar, bolsa rota $(9,6 \%)$. Já no pós-parto, os principais motivos foram: retenção placentária, com 5,8\% e hemorragia pós-parto e elevação de PA, ambas com 3,8\% (MENDES AS, et al., 2017).

Também caracterizou-se os RN das mulheres transferidas quanto ao peso e apgar, onde $76,3 \%$ (123) pesaram entre $2500 \mathrm{~g}$ e $4000 \mathrm{~g}$ e 1,8 e $7,4 \%$ foram menores que $2500 \mathrm{~g}$ e maior que $4000 \mathrm{~g}$, respectivamente. Em $14,2 \%$ não foi obtida essa informação. Se falando em apgar, $75,7 \%$ tiveram um apgar maior ou igual a sete. 
Tabela 3 - Motivos de transferências das gestantes e desfecho dos partos, $n=161$.

\begin{tabular}{|c|c|c|}
\hline Motivos de Transferências das Gestantes e Desfecho dos Partos & № & $\%$ \\
\hline \multicolumn{3}{|l|}{ Intraparto } \\
\hline Mecônio & 14 & 8,6 \\
\hline Distócia de progressão & 14 & 8,6 \\
\hline Síndromes hipertensivas & 12 & 7,4 \\
\hline Alteração de BCF & 11 & 6,8 \\
\hline Febre intraparto & 4 & 2,4 \\
\hline Sífilis & 3 & 1,8 \\
\hline ITU & 3 & 1,8 \\
\hline \multicolumn{3}{|l|}{ Pós-parto } \\
\hline Correção de laceração & 2 & 1,2 \\
\hline Febre pós-parto & 1 & 0,6 \\
\hline Curetagem & 1 & 0,6 \\
\hline Retenção placentária & 1 & 0,6 \\
\hline Motivos desconhecidos & 74 & 46,5 \\
\hline \multicolumn{3}{|l|}{ Desfecho do parto } \\
\hline Parto normal & 86 & 53,4 \\
\hline Cesárea & 60 & 37,2 \\
\hline Sem informação & 15 & 9,3 \\
\hline \multicolumn{3}{|l|}{ Características dos RNs } \\
\hline \multicolumn{3}{|l|}{ Peso } \\
\hline$<2500 \mathrm{~g}$ & 3 & 1,8 \\
\hline $2500 \mathrm{~g}$ a $4000 \mathrm{~g}$ & 123 & 76,3 \\
\hline$>4000 \mathrm{~g}$ & 12 & 7,4 \\
\hline Sem informação & 23 & 14,2 \\
\hline \multicolumn{3}{|l|}{ Apgar no primeiro minuto } \\
\hline $\begin{array}{l}<7 \\
>\text { ou igual a } 7\end{array}$ & $\begin{array}{c}13 \\
122\end{array}$ & $\begin{array}{c}8 \\
75,7\end{array}$ \\
\hline Sem informação & 26 & 16,1 \\
\hline
\end{tabular}

Fonte: Mendonça CPS, et al., 2021.

Percebe-se ainda o desfecho dos partos das gestantes transferidas, evidenciando que $53,4 \%$ resultaram em partos normais, enquanto $37,2 \%$ em cesariana. Pode-se observar que grande parte dos estudos evidenciou partos normais como o principal desfecho das remoções, sendo assim um achado semelhante ao presente estudo (53,4\%) (OLIVEIRA TCM, et al., 2019; BONADIO IC, et al., 2011; SILVA FMB, 2011). Porém nesta pesquisa, o número de cesarianas ainda se mostrou elevado, com uma taxa de $37,2 \%$, podendo ter relação com a cultura cesarista do Brasil, e com isso uma alta proporção de partos cirúrgicos no cenário obstétrico brasileiro. Outra possibilidade é a relação com as indicações de transferências, caracterizando reais complicações que necessitaram de partos cirúrgicos.

Neste contexto, a taxa de cesariana encontrada sobre o total de admissões no CPN foi de aproximadamente 10,2\%, estando dentro dos índices preconizados pela OMS, de 10 a 15\%. Pode-se então, afirmar a eficiência dos CPN como estratégia para redução da alta taxa de cesariana no Brasil, que já chegou a alcançar os mais altos índices do mundo em 2016, com mais de 55\% dos partos sendo via cesariana.

Essa taxa de intervenção cirúrgica após transferência poderia implicar no aumento da probabilidade do RN necessitar de cuidados neonatais mais complexos, porém neste estudo, $75,7 \%$ dos bebês de mães transferidas nasceram com o APGAR maior ou igual a 7. Em dois estudos encontrados, constatou-se que mais de $90 \%$ dos bebês de mães transferidas nasceram com apgar no primeiro minuto maior ou igual a 7 , e 
a média de peso foi entre 3268,33g e 3335g (MENDES AS, et al., 2017; BONADIO IC, et al., 2011). Esses dados podem indicar que a tomada de conduta no período apropriado garante um melhor prognóstico para os recém-nascidos. O índice de Apgar é uma medida usada para avaliação das condições de nascimento, porém, esse índice é limitado, e isoladamente, não indica cuidados necessários após o nascimento e prognóstico do RN.

No período estudado houve 425 partos normais no CPN, dos quais $44,7 \%$ dos bebês foram transferidos. Deve-se levar em consideração que alguns RN foram removidos objetivando uma avaliação do pediatra e parte deles voltava para o CPN, porém não se conseguiu obter a porcentagem dos bebês que retornaram e dos que foram internados.

Com relação ao percentual de transferências dos $\mathrm{RN}$ nascidos no CPN, o presente estudo mostrou um alto índice comparado à outras pesquisas apresentadas, que variaram entre $2 \%$ e 12,7\% (OLIVEIRA TCM, et al., 2019; LIMA DM, et al., 2018; PEREIRA ALF, et al., 2012; PEREIRA ALF, et al., 2013). Essa alta porcentagem pode ter se dado pelo grande quantitativo de bebês $(39,4 \%)$ terem sido transferidos para o alojamento conjunto sem registro de motivo, muitas vezes relacionado à necessidade de rotatividade do leito, não concluindo a alta hospitalar no setor.

Tabela 4 - Motivos de transferências dos RN e suas características de nascimento, $n=190$.

\begin{tabular}{lcc}
\hline Motivos de Transferência dos RN & № & $\%$ \\
\hline Icterícia & 28 & 14,7 \\
Desconforto respiratório & 25 & 13,1 \\
Acompanhar genitora transferida & 7 & 3,6 \\
Hipoglicemia & 2 & 1 \\
Sem informação & 120 & 63,1 \\
\hline Características de Nascimento dos RN Transferidos & № & $\%$ \\
\hline Sexo & \multicolumn{2}{c}{} \\
\hline Feminino & 99 & 47,3 \\
Masculino & 86 & 45,2 \\
Sem informação & 5 & 2,6 \\
\hline Peso ao nascer & \multicolumn{2}{c}{} \\
\hline <2500g & 2 & 1 \\
2500g a 4000g & 183 & 97,4 \\
> 4000g & 4 & 2 \\
Sem informação & 1 & 0,5 \\
\hline Apgar no 10 minuto & \multicolumn{3}{c}{} \\
\hline Maior ou igual a 7 & 176 & 92,6 \\
Menor que 7 & 12 & 6,3 \\
Sem informação & 2 & 1 \\
\hline
\end{tabular}

Fonte: Mendonça CPS, et al., 2021.

Não foram registrados os motivos das transferências de $63,1 \%$ dos RN. Porém, dentre os motivos listados, o mais prevalente é a icterícia do recém-nascido, em 14,7\%, seguida de desconforto respiratório (13,1\%). Uma porcentagem de 3,6\% RN foi transferida para acompanhar a genitora, 1\% por apresentar hipoglicemia neonatal, e as demais causas apresentaram $0,5 \%$, que são elas: necessidade de dieta orogástrica, hipóxia neonatal, corioaminionite, realização de exames, rastreio de sífilis, conjuntivite neonatal, baixo peso e intubação (Tabela 4).

O estudo realizado por Oliveira (2019) mostrou que 59,2\% RN foram removidos para outro serviço/setor por desconforto respiratório (DR), tendo sido apontada como a principal causa. Já no estudo realizado por Lima DM, et al. (2018), o DR ficou em terceiro lugar (3,4\%), sendo precedido de icterícia (46\%) e bebês não adequados para a idade gestacional $(11,5 \%)$. Uma pesquisa transversal e retrospectiva no Rio de Janeiro, teve um índice de remoção de $\mathrm{RN}$ de $6,6 \%$, e demonstrou os principais fatores como: DR $(30,6 \%)$, hipoatividade (17,3\%), baixo peso/prematuridade (16,3\%), infecção $(15,3 \%)$ e icterícia $(9,2 \%)$ (PEREIRA ALF, et al., 2012). 
17 RN necessitaram de reanimação neonatal, enquanto $173(91,1 \%)$ não necessitaram. Para quase $95 \%$ dos bebês foi proporcionado o contato pele a pele entre genitora de RN logo após seu nascimento. Foram pontuadas também as principais características dos RN que foram transferidos. Com relação ao sexo do bebê, os resultados de mostraram equivalentes, ou seja, $47,3 \%$ foram do sexo feminino e $45,2 \%$ do sexo masculino. Em 2,6\% não foram registradas essas informações. 97,4\% dos bebês pesaram entre 2500 e $4000 \mathrm{~g}$, e sobre o APGAR no $1^{\circ}$ minuto, $92,6 \%$ foi maior ou igual a 7 .

Observando um estudo realizado por Lima DM, et al. (2018) ao buscar as características dos RN transferidos, descobriu-se que $81,8 \%$ pesaram entre $2500 \mathrm{~g}$ e $4000 \mathrm{~g}, 9,8 \%$ pesaram mais que $4000 \mathrm{~g}$ e somente $8,4 \%$ pesou menos que $2500 \mathrm{~g}$ e em $98 \%$ dos RN o apgar no primeiro minuto de nascimento foi maior que 6. Outro estudo realizado por Oliveira TCM, et al. (2019) constatou que somente $2,7 \%$ dos RN tinham baixo peso. No presente estudo, a taxa de $\mathrm{RN}$ com baixo peso foi ainda inferior à taxa das pesquisas acima (1\%).

Tabela 5 - Características das mães dos RN transferidos e assistências prestada durante o TP, n=190.

\begin{tabular}{|c|c|c|}
\hline Características das Mães dos RN Transferidos & № & $\%$ \\
\hline \multicolumn{3}{|l|}{ Idade * } \\
\hline 10 a 19 anos & 44 & 23,1 \\
\hline 20 a 35 anos & 135 & 71 \\
\hline$>35$ anos & 9 & 4,7 \\
\hline Sem informação & 2 & 1 \\
\hline \multicolumn{3}{|l|}{ № de consultas de PN * } \\
\hline$>=6$ consultas & 133 & 70 \\
\hline$<6$ consultas & 55 & 28,9 \\
\hline Sem informação & 2 & 1 \\
\hline \multicolumn{3}{|l|}{ Idade gestacional * } \\
\hline Termo precoce & 52 & 27,3 \\
\hline Termo completo & 129 & 67,8 \\
\hline Termo tardio & 9 & 4,7 \\
\hline Assistência Prestada a Mãe do RN Durante TP e Parto & № & $\%$ \\
\hline \multicolumn{3}{|l|}{ Amniotomia } \\
\hline Sim & 34 & 17,8 \\
\hline Não & 156 & 82,1 \\
\hline \multicolumn{3}{|l|}{ Ocitocina } \\
\hline Sim & 35 & 18,4 \\
\hline Não & 155 & 81,5 \\
\hline \multicolumn{3}{|l|}{ Clampeamento do cordão } \\
\hline Imediato & 27 & 14,2 \\
\hline Tardio & 163 & 85,7 \\
\hline \multicolumn{3}{|l|}{ Reanimação } \\
\hline Sim & 17 & 8,9 \\
\hline Não & 173 & 91,1 \\
\hline \multicolumn{3}{|l|}{ Contato pele a pele } \\
\hline Sim & 11 & 5,7 \\
\hline Não & 179 & 94,2 \\
\hline
\end{tabular}

Fonte: Mendonça CPS, et al., 2021.

Tendo em vista o perfil das gestantes que tiveram seus recém-nascidos transferidos, com relação à idade, $23,1 \%$ mulheres foram consideradas adolescentes, $71 \%$ entre 20 a 35 anos e somente $4,7 \%$ foram mulheres maiores que 35 anos (Tabela 5).

Nota-se também que $70 \%$ das pacientes que tiveram seus bebês transferidos realizaram seis ou mais consultas de pré-natal. Tais transferências provavelmente não estão atreladas ao número de consultas, porém deve-se considerar a qualidade da assistência durante os atendimentos. Segundo Oliveira DM, et al. (2019) a inadequação da assistência pré-natal está relacionada com o aumento do risco materno e neonatal na população de gestantes brasileiras. O ministério da saúde recomenda no mínimo seis consultas de pré-natal 
para se obter uma assistência de qualidade, sendo realizados exames e orientações quanto a gestação, parto e pós-parto (BRASIL, 2012).

E em relação à idade gestacional, 52 mulheres $(27,3 \%)$ estavam no termo precoce, com idade gestacional entre 37semanas e 38sem e 6 dias, $129(67,8 \%)$ estavam no termo completo - entre 39 e 40 semanas e 6 dias, e 9 mulheres $(4,7 \%)$ eram consideradas no termo tardio, ou seja, maior que 41 semanas. Todas as pacientes que tiveram seus bebês transferidos pariram em uma gestação a termo. Tal taxa é caracterizada pelo protocolo clínico do serviço, onde só são admitidas pacientes à termo, sem nenhum tipo de comorbidade, com presença de exames pré-natais e em trabalho de parto ativo.

No que tange a assistência prestada à gestante durante seu trabalho de parto, observa-se que não foi realizada amniotomia em $82,1 \%$ das pacientes e não foi utilizada ocitocina em $81,5 \%$ delas. Cuidados recomendados pelo ministério da saúde como clampeamento tardio de cordão umbilical e contato pele a pele entre RN e genitora durante a hora dourada foram realizados em 85,7 e $94,2 \%$, respectivamente. De maneira geral, as características da assistência prestada não apresentam fatores que pudessem interferir de forma negativa nas condições de vitalidade do $\mathrm{RN}$, não podendo ter maiores relações, pois não foi investigada a indicação de fototerapia ou quantidade de dias de internamento. Ressalta-se que apenas foi averiguado que não houve nenhum internamento em cuidados intensivos por motivo de icterícia.

Resultados de recentes estudos mostram que o clampeamento tardio não apresenta associação significativa com a icterícia neonatal, apesar de demonstrar um risco relativo aumentado. Um estudo de 2017 relatou que entre dois grupos, um foi observado com clampeamento imediato e o outro tardio, as taxas de necessidade de fototerapia não foram maiores no grupo de clampeamento tardio (SOBIERAY NLEC, et al., 2019; GOES JF, 2017).

Durante o período de estudo, não foi constatada nenhuma morte materna e neonatal. É importante considerar que a assistência materna e neonatal é de responsabilidade das enfermeiras obstetras, e resultados favoráveis se deram devido à decisão das mesmas de transferirem em momento apropriado, sendo por precaução ou por risco iminente de agravamento do estado de saúde do binômio. Vale ressaltar que o trabalho no CPN é feito de forma interdisciplinar, contando com o apoio de médicos obstetras e neonatologistas quando necessário. Essa conduta visa garantir a continuidade do cuidado nas situações que é necessária uma assistência mais complexa. Porém, das 596 admissões, 72,5\% evoluíram de forma eutócica e foi assistenciado apenas pelas enfermeiras obstetras.

Contudo, os resultados aqui apresentados devem ser interpretados à luz das limitações presentes nesta pesquisa. A primeira, talvez a mais importante, está relacionada à fonte de dados. Por serem advindos de dados secundários obtidos por meio da análise dos registros, há uma importante incompletude, em sua grande maioria, pela falta de registros. Também deve-se considerar que tanto a planilha de registro das transferências maternas como livro de registro de RN não contemplavam alguns indicadores, sendo necessário buscar dados em livros de um outro setor, e como consequência, algumas informações estavam em falta.

Na interpretação dos resultados deste estudo é importante considerar fatores como a diferença entre casas de parto e CPN peri e intra-hospitalar, pois os critérios de admissão a transferências podem variar, e os modelos de assistência podem ser próprias de cada local. Deve-se considerar que tais limitações podem dificultar a precisão da realidade do serviço de saúde e por isso testifica-se a importância dos registros de forma correta e completa, e a necessidade de novas pesquisas que possam complementar este estudo.

\section{CONCLUSÃO}

A maioria das transferências foi realizada no período intraparto, primigestas, sem companheiro e com idade entre 20 e 35 anos. Os motivos das transferências não parecem estar relacionados com o tipo de assistência prestada no CPN e mais da metade dos desfechos resultaram em parto normal. Em relação aos recém-nascidos transferidos, a icterícia foi o motivo mais prevalente, podendo estar relacionada ao clampeamento tardio de cordão umbilical, porém não se pode afirmar tal fato. Apesar das taxas de 
transferência maternas e neonatais neste estudo serem consideradas maiores que nos estudos analisados, não houve nenhum agravamento ou óbito, e em relação ao total de admissões, a taxa de cesariana encontrouse dentro dos índices preconizados pela OMS, sugerindo que a assistência em CPN pode ser segura para as gestantes de risco habitual.

\section{REFERÊNCIAS}

1. ALBERS LL, KATZ VL. Birth setting for low-risk pregnancies. An analysis of the current literature. J Nurse Midwifery. 1991; 36: 215-20.

2. BONADIO IC, et al. Remoções maternas da Casa de Parto de Sapopemba para hospital de referência. Rev. Esc. Enferm USP 2011; 45(6): 1301-8.

3. BRASIL. Ministério da Saúde. Secretaria de Atenção á Saúde. Departamento de Ações Programáticas Estratégicas. Portaria № 1.459/GM, 24 de junho de 2011. Institui, no âmbito do Sistema Único de Saúde, a Rede Cegonha. Brasília (DF). 2011.

4. BRASIL. Lei no 7.498, de 25 de junho de 1986. Dispõe sobre a regulamentação do exercício da Enfermagem e dá outras providências. Brasília-DF; 1986.

5. BRASIL. Ministério da Saúde. Atenção ao pré-natal de baixo risco. Brasília: Ministério da Saúde. 2013.

6. BRASIL. Ministério da Saúde. Gestação de alto risco: Manual técnico. In: Estratégicas. 5 ed. Brasília, DF; 2012.

7. BRASIL. Portaria № 11, de 07 de Janeiro de 2015. Redefine as diretrizes para implantação e habilitação de Centro de Parto Normal (CPN), no âmbito do Sistema Único de Saúde (SUS), para o atendimento à mulher e ao recémnascido no momento do parto e do nascimento, em conformidade com o Componente parto e nascimento da Rede Cegonha, e dispõe sobre os respectivos incentivos financeiros de investimento, custeio e custeio mensal. Brasília: 2015.

8. CAMPOS SEV, LANA FCF. Resultados da assistência ao parto no Centro de Parto Normal Dr David Capistrano da Costa Filho em Belo Horizonte, Minas Gerais. Cad. Saúde Pública. 2007; 23(6): 1349-1359

9. CASTRO RCMB, et al. Resultados obstétricos e neonatais de partos assistidos. Rev enferm UFPE online, Recife, 12(4):832-9, abr., 2018.

10. FREITAS JMS, et al. Práticas obstétricas em centro de parto normal intra-hospitalar realizadas por enfermeiras obstetras. Esc Anna Nery 23(4) 2019.

11. GARCIA LV, et al. O centro de parto normal e sua contribuição para a atenção obstétrica e neonatal no Brasil. Revista Eletrônica Acervo Saúde. 2017; vol. Sup. 7, S356-S363.

12. GOES, JF. Clampeamento tardio do cordão umbilical: estudo de Coorte. 2017. 51 f. Dissertação (Mestrado em Ciências) - Fundação Oswaldo Cruz, Instituto Nacional de Saúde da Mulher da Criança e do Adolescente Fernandes Figueira, Rio de Janeiro, 2017.

13. KULIUKAS L, et al. Women's experience of intrapartum transfer from a Western Australian birth Centre co-located to a tertiary maternity hospital. BMC Pregnancy Childbirth. 2016;16:33.

14. LIMA DM, et al. Remoções neonatais do centro de parto normal Peri-hospitalar para o hospital. Rev Paul Enferm [Internet]. 2018; 29(1-2-3):47-55

15. MENDES AS, et al. Desfechos após transferência de um centro de parto normal para o hospital de referência. UDF centro universitário. 2017, 1(1); 1-11.

16. OLIVEIRA TCM, et al. Adequação da assistência pré-natal em casa de parto e causas associadas com as transferências hospitalares. Rev Gaúcha Enferm. 2019; 40: e20180419.

17. ORGANIZAÇÃO MUNDIAL DA SAÚDE. Maternidade segura. Assistência ao parto normal: um guia prático. Genebra: OMS; 1996.

18. PEREIRA ALF, et al. Assistência materna e neonatal na casa de parto David Capistrano filho, Rio de Janeiro, Brasil. R. pesq.: cuid. Fundam. Online 2012. Abr./jun. 4(2): 2905-13.

19. PEREIRA ALF, et al. Resultados maternos e neonatais de assistência em casa de parto no município do Rio de Janeiro. Esc Anna Nery 2013; 17 (1): 17-23.

20. RODRIGUES ARM, et al. Gravidez de alto risco: análise dos determinantes de saúde. Revista de Políticas Públicas, 2017. 16 (1); 23-28.

21. SANDALL J, et al. Midwife-led continuity models versus other models of care for childbearing women. Cochrane Database of Systematic Reviews 2016, Issue 4. Art. No.: CD004667.

22. SANTANA AT, et al. Atuação de enfermeiras residentes em obstetrícia na assistência ao parto. Rv Bras. Saúde Mater. Infant., 2019, 19(1): 145-155.

23. SILVA FMB, et al. Factors associated with maternal intrapartum transfers from a freestanding birth centre in São Paulo, Brasil: A case control study. Midwifery 28 (2012) 646-652.

24. SILVA FMB. Transferências maternas de uma casa de parto para o hospital: estudo caso-controle [dissertação]. São Paulo. Escola de Enfermagem da Universidade de São Paulo; 2011.

25. SOBIERAY NLEC, et al. Relação entre o tempo de clampeamento do cordão umbilical e incidência de Icterícia Neonatal e níveis de hematócrito em recém-nascidos a termo saudáveis. Arq. Med. Hosp. Fac. Cienc. Med. Santa Casa São Paulo, 2019; 64(2), 88-92.

26. TEIXEIRA CS, et al. Resultados maternos e neonatais em centro de parto normal intra-hospitalar. Enferm Foco 2018; 9 (1): 71-75.

27. VICO AF. Avaliação da implantação dos Centros de Parto Normal no Sistema único de Saúde. Dissertação (Mestrado em Ciências) - Instituto Nacional de Saúde da Mulher, da Criança e do Adolescente Fernandes Figueira, Rio de Janeiro - RJ, 2017; 81f. 